\title{
Les lésions musculaires des membres inférieurs : facteurs de risque et stratégies préventives
}

\section{Lower limb muscle injuries: Risk factors and preventive strategies}

\author{
F. Delvaux*, J.-F. Kaux, J.-L. Croisier
}

Département des sciences de la motricité, université, CHU de Liège, ISEPK, B21, allée des Sports 4, 4000

Liège, Belgique

Reçu le 28 mai 2015 ; accepté le 14 novembre 2016

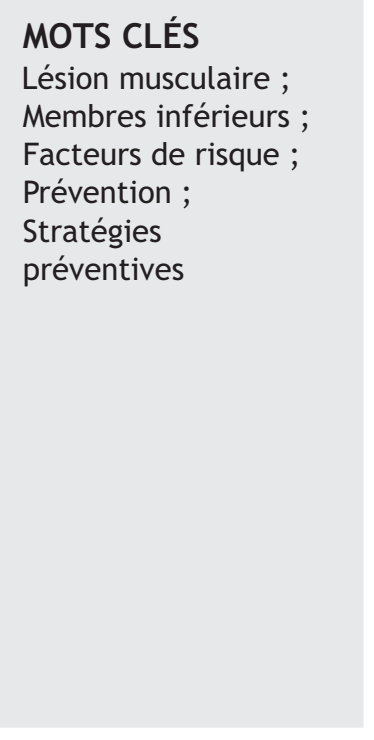

\begin{abstract}
Résumé
Objectifs. - L'incidence des lésions musculaires est élevée dans les sports où la vitesse de mouvement, l'explosivité ou les changements brusques de direction sont déterminants. Cet article a pour objectifs d'analyser : (1) les facteurs de risque des principales lésions musculaires des membres inférieurs (ischiojambiers, quadriceps, adducteurs et triceps sural) ; (2) l'efficacité des principales stratégies préventives de ce type de blessures.

Actualités. - Ces dernières années, un nombre significatif d'études prospectives a permis d'identifier plusieurs facteurs de risque de lésions musculaires. Parmi ceux-ci, un premier épisode lésionnel représente le seul facteur de risque intrinsèque retrouvé pour l'ensemble des quatre grands groupes musculaires. L'âge et la présence d'un déséquilibre musculaire interviennent plus spécifiquement dans la lésion des ischiojambiers ; la dominance dans la lésion du quadriceps ; un déséquilibre musculaire et un manque de souplesse dans la lésion des adducteurs, et enfin l'âge ainsi qu'un manque de souplesse dans la lésion du triceps sural. Les facteurs de risque extrinsèques apparaissent plus difficiles à associer à une localisation précise et sont principalement le type de sport pratiqué (sports collectifs et athlétisme notamment), un échauffement inadapté, une charge de travail mal répartie, ainsi que la pratique de l'activité en compétition. D'un point de vue préventif, il apparaît essentiel de favoriser la pratique régulière d'exercices de renforcement à haute intensité en mode excentrique et de corriger les éventuels déséquilibres musculaires.
\end{abstract}

* Auteur correspondant.

Adresse e-mail : fdelvaux@ulg.ac.be (F. Delvaux). 


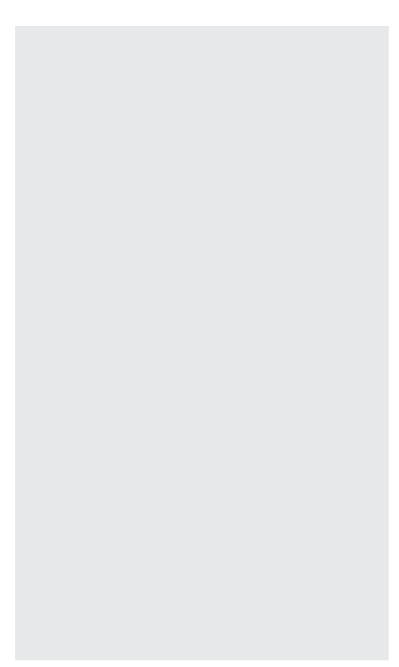

\section{KEYWORDS}

Muscle injury;

Lower limbs;

Risk factors;

Prevention;

Preventive strategies
Perspectives et projets. - L'influence de certains facteurs de risque comme le manque de souplesse dans les lésions des ischiojambiers et du quadriceps, la fatigue ou encore le moment de la saison mériterait d'être approfondie. La réalisation d'étirements, largement répandue sur les terrains de sport, ne devrait pas être remise en cause mais l'évidence scientifique de l'efficacité préventive de toute lésion musculaire reste à démontrer, et des précisions concernant les modalités pratiques des étirements (durée, type d'étirement...) sont souhaitables. Des études ultérieures sont également nécessaires pour confirmer l'intérêt préventif, dans les lésions des ischiojambiers et du quadriceps, de la thérapie manuelle ou d'exercices de gainage. Conclusion. - Un premier épisode de blessure musculaire représente le principal facteur de risque de lésions musculaires aux membres inférieurs, et chaque groupe musculaire semble présenter des facteurs de risque spécifiques à leur localisation. Pour les quadriceps et surtout pour les ischiojambiers, l'incidence lésionnelle peut être réduite de manière conséquente grâce à l'implémentation de certaines mesures comme la réalisation régulière d'exercices à haute intensité en mode excentrique.

๔ 2017 Elsevier Masson SAS. Tous droits réservés.

\section{Summary}

Objectives. - Muscle injuries are common in sports that involve high-speed and explosive movements or rapid changes in direction. The objectives of this article are: (1) to examine the risk factors for the main lower limb muscle strain-type injuries (hamstrings, quadriceps, adductors and triceps surae); (2) to analyze the effectiveness of preventive strategies for muscle injuries. News. - These last years, a significant number of prospective studies identified several risk factors for muscle injuries. The most important intrinsic risk factor, whatever the injury site, is a previous injury. Muscle imbalances and age specifically concern hamstring tears; dominance (kicking leg) predisposes to a quadriceps injury; muscle imbalances and a lack of flexibility concern adductors injury; age and a lack of flexibility are also risk factors for triceps surae injury. The lack of flexibility remains controversial in the literature. The main extrinsic risk factors are the type of sport, an insufficient warm-up, an unbalanced training load and sport during competition. Preventively, it appears essential to propose progressive high-intensity eccentric exercises and to correct potential muscle imbalances.

Prospects and projects. - The role of some risk factors such as: a lack of flexibility (specifically for hamstring and quadriceps injury), fatigue or the moment of the season should be cleared. Stretching activities should be continued but scientific evidence of its effectiveness for muscle injury prevention still needs to be demonstrated. There is also a need of evidence-based recommendations for optimal stretching modalities (duration, type of stretching...). Further studies are required to confirm the preventive interest, for hamstring and quadriceps tears, of manual therapy and core stability.

Conclusion. - A previous injury represents the most important risk factor for muscle injuries. Additionally, every muscle group of the lower limbs seems to present specific risk factors. Hamstring and quadriceps strain incidence may show a powerful decrease thanks to the implementation of preventive strategies such as high intensity eccentric exercises.

(c) 2017 Elsevier Masson SAS. All rights reserved.

\section{Introduction}

Les lésions musculaires représentent un type de blessure particulièrement fréquent chez le sportif, quel que soit le niveau. Récemment, une étude épidémiologique menée parmi des équipes professionnelles de football a montré qu'un club composé de 25 joueurs peut s'attendre à 15 lésions musculaires par saison, pouvant générer une moyenne totale de 223 jours d'absence des terrains, ainsi que 148 sessions d'entraînement et 37 matches officiels manqués [1]. Lors des dernières plus grandes compétitions mondiales d'athlétisme, les lésions musculaires sont apparues comme le type de blessure le plus fréquemment rencontré, tant chez les hommes que chez les femmes [2].
Dans leur immense majorité, elles touchent le membre inférieur, et plus particulièrement les muscles polyarticulaires soumis à des tensions importantes, notamment en mode de contraction excentrique [3].

À l'exception de certains sports à la gestuelle très spécifique (hockey sur glace par exemple), les ischiojambiers sont les muscles les plus souvent atteints. Ainsi, en football, 37 à $54 \%$ des blessures musculaires toucheraient les ischiojambiers $[1,4]$ et celles-ci représenteraient 10 à $23 \%$ des blessures totales [5]. La lésion des ischiojambiers n'est pas le privilège du football : l'incidence lésionnelle s'avère également élevée en athlétisme, rugby, handball ou encore cricket [6]. Durant ces activités sportives, la répétition de courses antérogrades à très haute vélocité 
implique, en fin de phase oscillante, la combinaison de mouvements brusques de flexion de hanche et d'extension de genou : les ischiojambiers se retrouvent ainsi, lors de chaque cycle complet de foulée, en allongement maximal et doivent fournir une contraction excentrique de haute intensité [7]. La lésion du muscle quadriceps, en particulier le droit fémoral $[8,9]$, apparaît aussi régulièrement dans les disciplines nécessitant des gestes répétés de sprints et de shoots comme le football $[10,11]$. Dix-neuf pour cents de l'ensemble des blessures musculaires survenant dans ce sport affecteraient le quadriceps et l'absence compétitive suite à cette lésion serait supérieure à celle des ischiojambiers [1]. Les adducteurs de hanche sont fortement sollicités en football, rugby, football australien ou natation, mais c'est surtout le hockey sur glace qui semble le plus traumatisant pour ces muscles : $10 \%$ des blessures totales et $43 \%$ des blessures musculaires atteignent les adducteurs [12]. L'incidence des lésions musculaires des adducteurs atteindrait 3,2 blessures pour 1000 heures de pratique en National Hockey League, championnat le plus relevé au monde dans cette discipline [13]. Dans ce sport, il a été suggéré que le mouvement de glissade, produit essentiellement par une contraction dynamique des abducteurs de hanche, place leurs antagonistes en position d'allongement potentiellement excessif. Les adducteurs ont donc pour objet de se contracter de manière excentrique afin de freiner ce mouvement violent d'abduction [13], ce qui favorise le développement de traumatisme musculaire intrinsèque. Enfin, si l'atteinte du triceps sural apparaît moins fréquente que celle des trois grands groupes musculaires cités cidessus, Ekstrand et al. [1] reportent cependant que $13 \%$ des blessures musculaires recensées sur des footballeurs professionnels concerne ce muscle.

Il apparaît également important de signaler que le taux de récidive des blessures musculaires est particulièrement élevé, surtout dans les sports à dimension explosive $[5,8]$.

Sur base de ces données épidémiologiques, il s'avère primordial de proposer des interventions préventives chez les athlètes exposés à ce risque lésionnel. Dans cette optique, il existe des modèles théoriques de stratégies préventives, comme celui développé par van Mechelen et al. [14] ou le modèle TRIPP (« Translating research into prevention practice ») : grâce à une démarche basée sur l'évidence scientifique, le respect des différentes étapes de ces modèles représentera ainsi un gage de qualité pour le praticien. Cependant, une étape essentielle et commune à ces deux modèles consiste à identifier l'ensemble des facteurs de risque des blessures. Or, si certains facteurs de risque ont été clairement identifiés comme favorisant avec certitude la lésion musculaire, d'autres revêtent un caractère plus spéculatif. De plus, selon le type de localisation ou le sport pratiqué, l'importance de chacun de ces facteurs peut varier dans des proportions parfois élevées : la connaissance de ces facteurs de risque permettra de préciser le(s) type(s) d'action(s) préventive(s) à mener. Ces mesures préventives, basées donc sur la correction d'un ou plusieurs facteurs de risque lésionnel avérés, devront ensuite remplir plusieurs conditions afin d'être réellement efficaces : être acceptées par l'ensemble du staff sportif et médical, assurer une compliance maximale vis-à-vis des athlètes, être mûrement réfléchies afin de ne pas développer d'autres facteurs de risque comme une surcharge, etc. Dès lors, la maîtrise de stratégies préventives qui ont fait la preuve de leur efficacité devrait pouvoir servir de modèle, non pas à dupliquer tel quel, mais à adapter intelligemment en fonction des spécificités des athlètes encadrés.

L'objectif de cet article consiste, d'une part, à analyser les facteurs de risque de lésion musculaire liée au sport et, d'autre part, à faire le point sur l'efficacité de diverses interventions à caractère préventif de ce type de lésion.

\section{Méthodologie}

Les articles recensés ont été sélectionnés via la base de données PubMed de 1985 à avril 2015. Deux séries de motsclés ont été combinées lors de la recherche : « risk factors, predisposing factors, prevention, preventive strategie(s), preventive program(s) » et «sports injury/injuries, muscle injury/injuries, muscle strain(s), hamstring strain/injury, adductor strain/injury, quadriceps strain/injury, rectus femoris strain/injury, calf strain/injury ». À partir des références bibliographiques trouvées, une recherche de proche en proche a ensuite été réalisée. Les études prospectives, les revues systématiques ainsi que les méta-analyses ont été conservées, tout comme les études prospectives originales. Pour que celles-ci soient conservées, le sujet abordé devait se référer à l'analyse de facteurs de risque ou de stratégies de prévention d'au moins l'une des blessures musculaires citées ci-dessus. Les avis d'experts, les rapports de cas ou d'une série de cas ainsi que les études rétrospectives n'ont pas été retenus. Les articles traitant de pathologies autres que concernant le tissu musculaire ont également été exclus (notamment les lésions tendineuses de type tendinopathie proximale du tendon des ischiojambiers, ou encore la pubalgie). Le flowchart de sélection d'articles est représenté sur la Fig. 1.

\section{Facteurs de risque lésionnels}

Classiquement, les facteurs de risque d'une pathologie se décomposent en deux catégories : les facteurs intrinsèques qui sont associés directement à une caractéristique propre au sujet, et les facteurs extrinsèques qui dépendent de paramètres externes à celui-ci. En complément, une autre classification en fonction du caractère modifiable ou non modifiable du facteur de risque peut également être envisagée. Le Tableau 1 reprend les facteurs de risque lésionnels avérés et spéculatifs en fonction de la topographie de l'atteinte (Tableau 1). Dans la suite de cet article, nous décrirons ces facteurs de risque de lésion musculaire selon l'atteinte des ischiojambiers, du quadriceps, des adducteurs de hanche ou du triceps sural.

\subsection{Lésion des ischiojambiers}

\subsubsection{Facteurs de risque intrinsèques}

3.1.1.1. Premier épisode de lésion. Selon plusieurs études réalisées parmi diverses disciplines collectives (football, football australien, rugby), 16 à $32 \%$ des lésions des ischiojambiers seraient en fait des récidives [1,15-17]. D'après Hagglund et al. [18], des footballeurs atteints d'une lésion musculaire des membres inférieurs lors d'une saison 


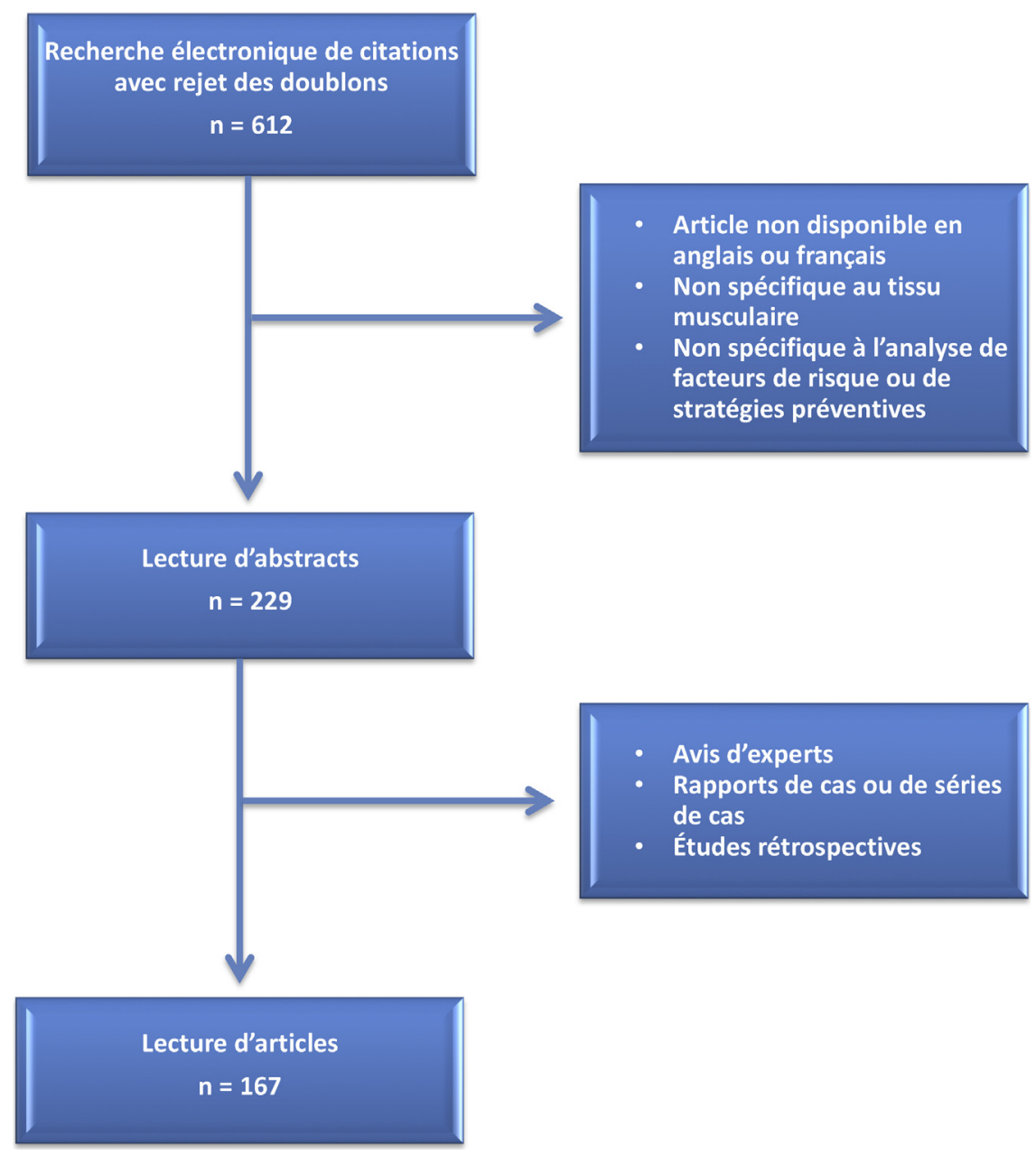

Figure 1 Flowchart de sélection d'articles.

présentent trois fois plus de chances de se blesser la saison suivante, comparativement à des joueurs non blessés. Des modifications structurelles du muscle ainsi qu'une insuffisance de correction des facteurs de risque de la première lésion expliquent probablement l'influence majeure de ce premier épisode lésionnel sur le risque ultérieur de rechute [7]. L'influence d'une déchirure des ischiojambiers sur le risque subséquent de s'occasionner une rupture du ligament croisé antérieur, et inversement, est aussi actuellement envisagée par certains experts [19].

3.1.1.2. Âge. De nombreuses études ont démontré que les sportifs plus âgés étaient plus exposés aux blessures musculaires, en particulier aux ischiojambiers [18,20,21]. Ainsi, en football, l'incidence lésionnelle semble déjà supérieure à partir de 23-24 ans [22,23] et serait multipliée par un facteur de 1,8 par année [22]. Les raisons pour lesquelles des athlètes plus âgés s'occasionnent plus facilement des lésions musculaires ne sont pas tout à fait claires. Il est souvent suggéré que des modifications associées à l'âge telles qu'une augmentation de l'indice de masse corporelle (IMC) ou une perte de souplesse musculotendineuse expliquent en partie le problème [24].

3.1.1.3. Déséquilibre de force musculaire. Plusieurs études ayant abordé le sujet identifient un manque de force des ischiojambiers comme facteur de risque lésionnel sur ces muscles $[25,26]$ chez des footballeurs, même si ce constat n'est pas entièrement partagé par tous [27]. Pour ces muscles, un déficit de force excentrique apparaît particulièrement impliqué [28] : un ratio mixte (moment de force maximale des ischiojambiers excentrique à $30 \%$ s/quadriceps concentrique à $240 \%$ s) a été identifié comme le paramètre le plus discriminant [26]. Yeung et al. [29] constatent, chez des sprinteurs, un risque multiplié par 17 lorsque le ratio de force ischiojambiers/quadriceps (en mode concentrique à une vitesse angulaire de $180^{\circ}$ par seconde) est inférieur à 0,60 . En synthèse, une asymétrie de force musculaire entre le côté gauche et le côté droit et/ou une faiblesse d'un muscle par rapport à son antagoniste semblent étroitement associés à un risque accru de blessure.

3.1.1.4. Manque de souplesse. La littérature fournit des résultats contradictoires à propos de ce paramètre : si certains auteurs ont mis en évidence une association entre manque de souplesse et lésion des ischiojambiers $[21,30,31]$, d'autres études n'établissent aucun lien direct entre souplesse et risque lésionnel [22,32,33].

3.1.1.5. Taille-poids-indice de masse corporelle. 丸̀ l'exception d'une étude qui a identifié qu'un IMC supérieur à 25 favoriserait la lésion des ischiojambiers et une augmentation de $1 \mathrm{~kg}$ de poids corporel augmenterait 
Tableau 1 Facteurs de risque avérés et spéculatifs des principales lésions musculaires des membres inférieurs.

\begin{tabular}{|c|c|c|}
\hline $\begin{array}{l}\text { Muscles } \\
\text { atteints }\end{array}$ & Facteurs de risque avérés & Facteurs de risque potentiels \\
\hline Ischiojambiers & $\begin{array}{l}\text { Premier épisode lésionnel } \\
\text { Âge } \\
\text { Déséquilibre de force } \\
\text { Pratique d'un sport explosif } \\
\text { Pratique en compétition } \\
\text { Échauffement inadapté }\end{array}$ & $\begin{array}{l}\text { Manque de souplesse } \\
\text { Taille-poids-IMC } \\
\text { Race } \\
\text { Dysfonction sacro-iliaque ou lombaire } \\
\text { Charge d'entraînement mal équilibrée } \\
\text { Fatigue } \\
\text { Position dans le jeu } \\
\text { Compétition à domicile }\end{array}$ \\
\hline Quadriceps & $\begin{array}{l}\text { Premier épisode lésionnel } \\
\text { Position dans le jeu } \\
\text { Dominance dans les sports avec gestes de shoot } \\
\text { Pratique d'un sport explosif } \\
\text { Pratique en compétition }\end{array}$ & $\begin{array}{l}\text { Âge } \\
\text { Déséquilibre de force musculaire } \\
\text { Manque de souplesse } \\
\text { Taille-poids-IMC } \\
\text { Échauffement inadapté } \\
\text { Charge d'entraînement mal équilibrée } \\
\text { Moment de la saison } \\
\text { Pratique sportive sur terrain sec }\end{array}$ \\
\hline Adducteurs & $\begin{array}{l}\text { Premier épisode lésionnel } \\
\text { Déséquilibre de force adducteurs/abducteurs } \\
\text { Position dans le jeu } \\
\text { Pratique d'un sport explosif } \\
\text { Échauffement inadapté }\end{array}$ & $\begin{array}{l}\text { Manque de souplesse } \\
\text { Compétition à domicile } \\
\text { Dominance }\end{array}$ \\
\hline Triceps sural & $\begin{array}{l}\text { Premier épisode lésionnel } \\
\text { Âge } \\
\text { Pratique d'un sport explosif } \\
\text { Position dans le jeu } \\
\text { Pratique en compétition } \\
\text { Manque d'échauffement }\end{array}$ & $\begin{array}{l}\text { Manque de souplesse } \\
\text { Dominance }\end{array}$ \\
\hline
\end{tabular}

de $7 \%$ ce risque lésionnel [23], les experts s'accordent globalement sur le fait qu'il n'existe pas de réel lien entre poids, taille ou encore IMC et risque de lésion musculaire des ischiojambiers [34].

3.1.1.6. Race/appartenance ethnique. L'origine ethnique aborigène ou africaine a été associée à un risque accru de lésion des ischiojambiers [23]. Bien que la cause ne soit formellement identifiée, il a été suggéré que, dans ces populations, une proportion plus élevée de fibres de type Il et une antéversion plus marquée du bassin soient impliqués en tant que facteurs favorisants $[23,35]$.

3.1.1.7. Dysfonction lombaire ou sacro-iliaque. Deux études ont suggéré qu'un épisode de lombalgie d'origine zygoapophysaire ou une dysfonction sacro-iliaque (notamment une antéversion de l'os iliaque) pourraient favoriser la survenue de lésion des ischiojambiers [36,37]. Cependant, l'évidence scientifique à ce propos ne peut qu'être qualifiée de faible et nécessiterait de futures recherches.

3.1.1.8. Dominance. Dans l'ensemble des disciplines sportives, les blessures aux ischiojambiers semblent distribuées de manière globalement équivalente entre les jambes dominante et non dominante [38].

3.1.1.9. Autres facteurs intrinsèques. Certains facteurs ont été associés à un risque accru de blessure capsuloligamentaire ou tendineux, mais n'ont jamais été rigoureusement associés à la pathologie ischiojambière ou musculaire au sens large : manque d'équilibre, faible contrôle neuromusculaire, pauvre qualité de gestuelle sportive, sexe, faible niveau d'endurance, facteurs psychosociaux, laxité ligamentaire ou encore troubles de statique.

\subsubsection{Facteurs de risque extrinsèques}

3.1.2.1. Type de sport. La pratique d'un sport à dimension explosive, comprenant des gestes de sprints courts et/ou longs, des sauts ou encore des changements brusques de direction favorise très clairement la survenue d'une lésion musculaire. Ces sollicitations soumettent la musculature des membres inférieurs, notamment en mode de contraction excentrique, à des forces de tension particulièrement élevées, propices à créer des lésions anatomiques sur l'élément musculaire [8]. Spécifiquement, l'incidence de la lésion des ischiojambiers apparaît particulièrement élevée en sprint et dans les sports collectifs tels le football [6].

3.1.2.2. Échauffement inadapté. La pratique d'un échauffement permet notamment d'améliorer l'extensibilité musculaire via des modifications des propriétés viscoélastiques [39]. Il apparaît donc logique qu'un muscle échauffé pourra non seulement développer un meilleur niveau de force, mais ne devrait également subir une lésion de type déchirure, qu'à un degré d'étirement supérieur [40]. À notre connaissance, aucune étude n'a étudié spécifiquement l'influence d'un échauffement sur la survenue de lésion aux ischiojambiers. 
3.1.2.3. Charge d'entraînement. Une mauvaise répartition de la charge de travail, un manque de progressivité lors de la reprise en période de présaison, ... : : un programme d'entraînement mal adapté semble contribuer à une augmentation des lésions musculaires [36,41,42]. De même, la réalisation d'exercices à haute intensité, surtout en mode excentrique et chez des athlètes peu familiarisés, pourrait induire des delayed onset muscular soreness (DOMS). De manière transitoire, ces courbatures, qui se manifestent par une perte de force et une raideur transitoires, placent les athlètes en situation de risque lésionnel majoré : la réalisation d'entraînements à intensité élevée durant cette période pourrait générer plus facilement des lésions musculaires [43]. Une mauvaise gestion de la charge d'entraînement n'a cependant jamais été associé de manière formelle avec une augmentation spécifique de l'incidence lésionnelle des ischiojambiers.

3.1.2.4. Fatigue. Plusieurs auteurs ont observé que la lésion des ischiojambiers survient plus souvent durant les dernières parties d'entraînement ou de compétitions $[1,16,39,41]$. Ceci suggère que la fatigue favorise la blessure musculaire en induisant des modifications physiologiques au sein du muscle, une diminution de la coordination, de la concentration ou de la gestuelle sportive [36]. Une étude animale avait mis en évidence qu'un muscle préfatigué était plus susceptible de subir une déchirure en raison d'une réduction de sa capacité à résister à l'allongement [44]. Chez l'humain, il a été démontré qu'une fatigue des ischiojambiers induite par des exercices répétés amenait une augmentation de l'amplitude d'extension du genou en phase ultime d'oscillation lors de la course à pied [45], ainsi qu'une réduction de la flexion de hanche [46]. Ces changements de la biomécanique de course pourraient donc induire des tensions excessives dans les ischiojambiers et ainsi favoriser le développement de lésions anatomiques sur ces derniers.

3.1.2.5. Contexte sportif. À l'instar d'une large majorité de blessures sportives, les lésions musculaires surviennent plus fréquemment lors de compétitions que lors d'entraînements $[1,18,47]$. En football professionnel, Ekstrand et al. relatent ainsi des taux de blessures musculaires, localisées préférentiellement aux ischiojambiers, six fois supérieurs en compétition par rapport à l'entraînement (8,70 vs 1,37 blessures/1000 heures d'exposition) [1].

3.1.2.6. Terrain de jeu. Curieusement, les blessures aux ischiojambiers dans le football professionnel apparaissent plus fréquentes lors de matches à domicile que lors de matches en déplacement [48] : les auteurs émettent l'hypothèse qu'en jouant à domicile, un taux supérieur de possession de balle combiné à un plus grand temps de jeu en zone d'attaque augmenterait le nombre de sprints et de passes. Ainsi, pour l'équipe qui reçoit, une intensité de jeu globalement supérieure favoriserait la survenue de lésions musculaires. Cependant, des facteurs sous-jacents comme un degré d'anxiété supérieur lors de rencontres à domicile pourraient aussi être impliqués [49].

3.1.2.7. Position dans le jeu. En football, les gardiens de but seraient moins exposés aux lésions musculaires, quelle que soit la localisation de la blessure (et ce compris l'atteinte des ischiojambiers) [49]. En rugby, il semble que les joueurs arrière s'occasionnent plus facilement des lésions des ischiojambiers [16].
3.1.2.8. Moment de la saison. Une distribution équilibrée de blessures aux ischiojambiers entre la présaison et la période compétitive a été observée par plusieurs auteurs $[50,51]$.

3.1.2.9. Autres facteurs extrinsèques. Enfin, les facteurs suivants sont régulièrement cités comme facteurs de risque lésionnels au sens large, mais l'évidence scientifique que ceux-ci jouent un rôle central dans la survenue de blessures musculaires en général ou aux ischiojambiers plus spécifiquement semble hautement spéculative : règlement sportif, niveau de formation des entraîneurs, équipement, prise de médicaments ou produits dopants, niveau de jeu, fair-play, temps de jeu...

\subsection{Lésion du quadriceps}

\subsubsection{Facteurs intrinsèques}

3.2.1.1. Premier épisode de lésion. Tout comme pour les ischiojambiers, un premier épisode de lésion représenterait un facteur de risque important de nouvelle lésion [49]. Le risque de lésion du quadriceps après avoir subi un épisode de lésion musculaire localisé sur un autre groupe musculaire semble également majoré : Hagglund et al. [49] ont démontré qu'une blessure au mollet ou aux adducteurs favorise la lésion du quadriceps. Orchard et al. [51] signalaient également qu'une lésion aux ischiojambiers majorerait le risque de blessure sur le quadriceps : une biomécanique de course modifiée suite à la blessure aux ischiojambiers (notamment une réduction de la longueur du pas) pourrait en effet protéger ces muscles mais augmenter les chances de lésion au quadriceps.

3.2.1.2. Âge. Trois larges études prospectives n'ont pas montré de lien entre lésion du quadriceps et âge $[1,30,52]$. 3.2.1.3. Déséquilibre de force musculaire. Fousekis et al. [52] ont observé une tendance, non significative cependant, vers une plus faible incidence de lésion du quadriceps chez les footballeurs qui ne présentaient pas d'asymétrie de force excentrique sur ce muscle. Dans cette étude, le manque de résultat statistiquement significatif était attribué au faible nombre de lésions du quadriceps survenues dans la population (7 lésions du quadriceps pour 100 footballeurs).

3.2.1.4. Manque de souplesse. L'étude de Witvrouw et al. [31] ont constaté, chez des footballeurs de haut niveau, qu'un manque de souplesse du quadriceps favorise la lésion de ce muscle.

3.2.1.5. Taille-poids-indice de masse corporelle. Orchard et al. ont établi, chez des footballeurs australiens, un lien entre risque lésionnel sur le quadriceps et une taille inférieure à $182 \mathrm{~cm}$ [51], sans entrevoir cependant d'explication rationnelle à ce constat.

3.2.1.6. Dominance. Les lésions du quadriceps semblent apparaître plus fréquemment du côté de la jambe de shoot en football et en football australien (minimum $60 \%$ ) $[1,51]$.

\subsubsection{Facteurs extrinsèques}

3.2.2.1. Contexte sportif. Un footballeur élite semble présenter moins de risque de se blesser au quadriceps lors d'un match de Champion's League que lors d'une rencontre nationale [49]. Les auteurs de l'étude concèdent que l'explication de ce constat reste peu claire. 


\subsubsection{Terrain de jeu. Orchard et al. [53] et Woods et al.} [50] ont remarqué une incidence plus élevée de lésions du quadriceps lors de la pratique du football sur terrain sec ou lorsque les pluies ont été peu abondantes la semaine précédant le match.

3.2.2.3. Position dans le jeu. Les gardiens de but en football présentent un risque inférieur de lésion quadricipitale en comparaison avec les joueurs de champs [49].

3.2.2.4. Moment de la saison. Le début de saison semble générer une proportion supérieure de blessures au quadriceps chez le footballeur $[48,50]$.

\subsection{Lésion des adducteurs}

\subsubsection{Facteurs intrinsèques}

3.3.1.1. Premier épisode de lésion. Hagglund et al. [49] identifient, chez des footballeurs de haut niveau, un premier épisode de lésion des adducteurs comme prédictif de récidive. Selon Tyler et al., le taux de récidive de lésion des adducteurs grimperait, chez des joueurs élites en hockey sur glace, jusqu'à $44 \%$ [13]. En football australien, l'incidence de récidive apparaît également élevée : $32 \%$ des nouvelles lésions des adducteurs seraient effectivement des rechutes [53]. Si ces deux dernières études ne permettent pas d'affirmer qu'un premier épisode lésionnel influence directement la survenue d'une récidive (méthodologie non prospective), ces taux particulièrement élevés de récidive amènent à se questionner sur un lien entre ces différents épisodes lésionnels.

3.3.1.2. Déséquilibre musculaire. Dans son étude sur le hockey professionnel, Tyler et al. [13] relatent également un risque de lésion des adducteurs multiplié par 17 lorsque la force des adducteurs de hanche n'atteint pas $80 \%$ de la force des abducteurs correspondants. Toujours parmi une population de hockeyeurs sur glace, deux études sur les trois recensées par Hrysomallis [54] ont démontré un lien entre asymétrie de force des muscles adducteurs et risque lésionnel.

3.3.1.3. Manque de souplesse. Trois études sur quatre ayant recherché un lien, dans le monde du football, entre manque de souplesse des adducteurs et risque lésionnel de ces muscles, ont effectivement trouvé une relation positive entre ces deux paramètres $[20,31,55,56]$. Par contre, en hockey sur glace ou en rugby, le manque de souplesse des adducteurs ne semble pas s'avérer prédictif d'une blessure ultérieure $[13,57,58]$.

3.3.1.4. Dominance. En football professionnel, le côté dominant apparaît plus fréquemment atteint (56 \%) que le côté non dominant [49].

\subsubsection{Facteurs extrinsèques}

3.3.2.1. Position dans le jeu. Les gardiens de but en football présentent un risque inférieur de lésion des adducteurs en comparaison avec les joueurs de champs [49].

3.3.2.2. Terrain de jeu. Dans leur suivi de matches de Champion's League de football sur plusieurs années, Hagglund et al. ont constaté que les adducteurs, à l'instar des ischiojambiers, étaient plus fréquemment atteints lors de matches à domicile que lors de matches en déplacement [48] (voir hypothèse des auteurs au Section 3.1.2.6).

\subsection{Lésion du triceps sural}

\subsubsection{Facteurs intrinsèques}

3.4.1.1. Premier épisode lésionnel. En football, la survenue d'une blessure au triceps sural a été associée à un premier épisode de blessure sur ce même groupe musculaire. Il est intéressant de noter qu'une lésion sur les adducteurs ou sur les ischiojambiers majore également le risque de lésion musculaire du mollet [49].

3.4.1.2. Âge. Dans une étude récente, Ekstrand et al. ont constaté un lien étroit entre l'âge de footballeurs professionnels et l'incidence de lésion du triceps sural : de 0,32 blessures par 1000 heures de pratique parmi les 16-21 ans, l'incidence augmente à 1,07 chez les 22-30 ans, et atteint un sommet chez les plus de 30 ans avec 1,89 [1]. Hagglund et al. ont constaté un risque lésionnel doublé chez les footballeurs élites plus âgés, comparativement aux plus jeunes [49].

3.4.1.3. Manque de souplesse. Le manque de souplesse du triceps sural n'a pas été identifié comme facteur de risque de lésion sur ce muscle dans l'étude de Witvrouw et al. parmi une population de footballeurs élites [31].

3.4.1.4. Dominance. Aucun effet de dominance n'a été mis en évidence parmi une large population de footballeurs professionnels étudiée par Hagglund et al. [49].

\subsubsection{Facteurs extrinsèques}

3.4.2.1. Position dans le jeu. Tout comme pour les groupes musculaires précédemment décrits dans cet article, les gardiens de but de football semblent présenter un risque lésionnel réduit [49].

3.4.2.2. Contexte sportif. À l'opposé des lésions quadricipitales, la participation à la Champion's League de football semble majorer le risque de blessure du triceps sural comparativement à la participation aux rencontres nationales [49] : un niveau de jeu et/ou d'anxiété supérieur pourrait expliquer ce constat.

\section{Stratégies préventives}

Deux logiques peuvent s'opposer lorsque l'on entreprend une démarche préventive : soit l'ensemble des sportifs réalisent un programme identique, il s'agit ainsi d'une démarche groupée $[59,60]$; soit chaque athlète réalisera un programme «sur mesure » basé sur sa propre exposition aux blessures : c'est la démarche individualisée [26].

La démarche groupée présente comme principaux avantages d'être relativement simple à mettre en place et moins coûteuse en termes de temps d'évaluation et en termes de budget. Par contre, cette démarche peut s'avérer insuffisante chez un athlète présentant un risque (très) élevé de blessure avec des déficiences importantes : le programme préventif pourrait être trop peu intense pour ce dernier et le risque lésionnel resterait grand. La démarche individualisée permet, sur base d'évaluations pertinentes, de cibler plus précisément les paramètres à améliorer afin de réduire le risque lésionnel. L'efficacité de cette démarche est donc généralement supérieure à la démarche groupée. Par contre, la démarche individualisée nécessite un investissement plus important en termes de temps, d'organisation et de budget (Tableau 2). 
Tableau 2 Synthèse des différentes stratégies préventives.

\begin{tabular}{|c|c|c|}
\hline Mesure préventive & Localisation & Paramètre d'intervention \\
\hline \multirow[t]{2}{*}{ Correction de déséquilibre musculaire } & Ischiojambiers [26] & $\begin{array}{l}\text { Ratios de force ischiojambiers/quadriceps } \\
\text { Comparaison bilatérale }\end{array}$ \\
\hline & Adducteurs [62] & Ratios de force adducteurs/abducteurs \\
\hline \multirow[t]{2}{*}{ Renforcement musculaire en mode excentrique } & Ischiojambiers & Nordic Hamstring Exercise \\
\hline & {$[16,59,60,64]$} & Reverse Nordic Hamstring Exercise \\
\hline Thérapie manuelle & Ischiojambiers [69] & Mobilisations, Manipulations \\
\hline Amélioration de la souplesse & NS & NS \\
\hline $\begin{array}{l}\text { Gestion optimale de la charge de travail } \\
\text { Correction d'une mauvaise gestuelle sportive } \\
\text { Réalisation d'exercices de core stability }\end{array}$ & NS & NS \\
\hline
\end{tabular}

\subsection{Correction de déséquilibres musculaires}

\subsubsection{Ischiojambiers}

Dans son étude de 2008, Croisier et al. [26] ont observé que la correction de déséquilibres de force des ischiojambiers présents chez des footballeurs de haut niveau $(n=462)$ ramenait ces sportifs à un risque lésionnel quasi égal à ceux ne présentant pas de déséquilibre, alors qu'au départ ce risque était plus de quatre fois supérieur. Il est intéressant de noter que les footballeurs avec déséquilibre musculaire mais qui n'avaient pas réalisé de renforcement subséquent ou bien qui avaient réalisé un programme de renforcement mais sans évaluation isocinétique de contrôle, ont montré des taux de blessures aux ischiojambiers significativement plus élevés.

Par ailleurs, la prévention de récidive après un premier épisode de lésion des ischiojambiers nécessite prioritairement un recouvrement optimal des qualités musculaires [28]. Dès lors, la réalisation d'une évaluation isocinétique avant de décider le retour sur terrain d'un athlète blessé s'avère indispensable. Ce constat est partagé par l'ensemble des médecins responsables de clubs français et belges de football professionnel [61].

\subsubsection{Adducteurs}

Tyler et al. ont réalisé une étude interventionnelle chez des hockeyeurs sur glace [62]. Trente-trois joueurs qui présentaient des ratios de force adducteurs/abducteurs inférieurs à $80 \%$, soit considérés comme présentant un risque majoré de lésion des muscles adducteurs, ont subi un programme de remédiation jusqu'à atteindre ce seuil. Ce programme était composé d'exercices de renforcement des muscles adducteurs et était effectué sous la forme d'ateliers de musculation combinés à des exercices spécifiques de terrain. Une très nette réduction de l'incidence des lésions musculaires des adducteurs (incidence lésionnelle divisée par 4,5) a été observée sur les deux saisons suivantes. Tout comme l'étude de Croisier et al. [26], cette étude suggère fortement qu'une réduction significative du risque de blessure musculaire pourrait être atteinte grâce à la détection et la correction de déséquilibres musculaires.

\subsection{Renforcement musculaire en mode excentrique}

\subsubsection{Ischiojambiers}

L'efficacité d'exercices de renforcement musculaire en mode excentrique en tant qu'outil de prévention primaire a surtout été étudiée sur les ischiojambiers [63]. Récemment, une large étude hollandaise [59] a démontré l'intérêt d'implémenter le Nordic Hamstring Exercise (NHE) chez des footballeurs amateurs. Cet exercice consiste, à partir d'une position à genoux redressé et les pieds fixés au sol grâce à l'aide d'un partenaire, à se pencher lentement vers l'avant en freinant la descente, ce qui implique une contraction excentrique importante des ischiojambiers. Dans cette étude randomisée et contrôlée, le groupe réalisant de manière régulière cet exercice a présenté une incidence de lésions des ischiojambiers significativement inférieure (11 blessures versus 25 blessures dans le groupe témoin) [59]. Si cette étude est probablement la première à avoir étudié l'impact positif du NHE sur l'incidence lésionnelle chez des sportifs amateurs, plusieurs recherches avaient déjà mis en évidence son efficacité chez des sportifs de haut niveau $[16,60,64]$.

Arnason et al. [64] ont ainsi observé que les équipes professionnelles de football qui incluent le NHE dans leurs entraînements réduisaient l'incidence de lésion des ischiojambiers de $65 \%$, comparativement aux équipes qui ne l'utilisaient pas. De plus, lorsque l'on comparait avec les saisons précédentes, l'incidence ainsi que la sévérité des blessures aux ischiojambiers était significativement réduite au sein des équipes qui ont utilisé le NHE. De même, parmi une très large cohorte de footballeurs de haut niveau, Petersen et al. [60] ont constaté une incidence de lésion des ischiojambiers significativement très inférieure dans le groupe « intervention avec NHE » (15 blessures) par rapport au groupe témoin (52 blessures). Chez des rugbymen anglais professionnels, Brooks et al. [16] remarquent aussi que ceux qui utilisent le NHE en plus de leur programme classique de renforcement présentent moins de hamstring strains que ceux qui ne le pratiquent pas.

Pour obtenir de bons résultats en termes de prévention lésionnelle, la compliance des sujets doit être maximale : 
à côté des études décrites ci-dessus qui ont montré d'excellents résultats grâce à la pratique du NHE, d'autres recherches n'ont pas retrouvé cette efficacité $[22,47]$. Mais dans ces dernières, la compliance était loin d'être optimale et, selon les auteurs, est probablement à l'origine de ce constat d'échec.

\subsubsection{Quadriceps et autres localisations}

À l'instar du NHE pour les ischiojambiers, il existe de nombreux exercices de renforcement en mode de contraction excentrique pour d'autres groupes musculaires. Il semble raisonnable de penser que ceux-ci pourraient s'avérer efficaces préventivement, mais la littérature apparaît extrêmement pauvre dès qu'il s'agit d'autres muscles que les ischiojambiers.

Un protocole préventif de renforcement excentrique du quadriceps a été mis en place par Brughelli et al. [65] chez 13 footballeurs professionnels. Ceux-ci n'ont présenté aucune lésion subséquente du quadriceps, tandis que deux lésions du droit antérieur, objectivées par IRM, sont apparues dans le groupe témoin $(n=11)$. Les exercices réalisés comprenaient notamment le Reverse Nordic Hamstring qui consiste, en partant d'une position à genoux redressé et hanches en extension, à se laisser pencher vers l'arrière et solliciter ainsi une contraction importante des quadriceps en mode excentrique.

En synthèse, Mendiguchia et al. [43] recommandent de suivre les principes suivants dans le choix des exercices: travail en chaînes cinétiques ouverte et fermée, implication bilatérale et unilatérale, implication de plusieurs articulations, facilité de mise en place, et intégration d'exercices excentriques proches du sport pratiqué par le sujet. Nous pourrions compléter ces recommandations en préconisant une progression vers des exercices réalisés à (très) haute intensité afin de favoriser le développement de la force excentrique maximale.

\subsection{Amélioration de la souplesse}

À notre connaissance, aucune étude n'a formellement démontré l'efficacité préventive d'étirements sur l'incidence de lésions musculaires. Ceci n'apparaît pas forcément étonnant vu la difficulté préalable d'identifier en amont le manque de souplesse comme facteur de risque lésionnel [31,55]. Pope et al. [66] relatent bien des taux inférieurs de lésions musculaires au niveau de la cuisse chez des athlètes pratiquant des étirements par rapport à un groupe témoin (différence significative). Mais dans cette étude, la durée des étirements (20 s par groupe musculaire), insuffisante que pour obtenir une modification de la compliance musculaire, implique d'interpréter prudemment ces résultats [67].

Une majorité d'experts considère néanmoins que la pratique régulière d'étirements représente un élément préventif important. L'article de McCall et al. [68] reflète parfaitement ce constat : dans cette étude, les médecins responsables des équipes nationales de football présentes à la dernière coupe du monde au Brésil étaient notamment interrogés sur les facteurs de risque et les stratégies préventives qu'ils percevaient comme les plus importants. $\mathrm{Si}$ le manque de souplesse ne figurait pas parmi les cinq facteurs de risque intrinsèques les plus essentiels, la pratique d'étirements représentait la seconde méthode préventive la plus importante à leurs yeux.

À ce stade, il apparaît donc raisonnable de recommander la pratique d'étirements. Mais des informations précises et concrètes concernant la durée, le moment, l'intensité ou encore le type d'étirements optimaux (statique, contracter-relâcher, dynamique...) restent toutefois lacunaires et nécessitent des explorations ultérieures.

\subsection{Autres stratégies préventives possibles}

\subsubsection{Thérapie manuelle}

Dans une revue systématique Cochrane parue en 2010 sur la prévention des lésions des ischiojambiers [69], les auteurs relatent une étude australienne (données non publiées) intéressante. Celle-ci a exploré l'intérêt de réaliser préventivement des sessions de thérapie manuelle chez des joueurs de football australien. Pour chaque sujet du groupe expérimental $(n=28)$, différentes techniques pouvaient être appliquées durant la saison, telles des manipulations à haute fréquence et basse amplitude ou encore des mobilisations des tissus mous. Les résultats ont montré que le groupe expérimental a développé significativement moins de blessures musculaires des ischiojambiers que le groupe témoin. Bien que la cohorte utilisée dans cette étude est relativement faible et que cette étude n'a étrangement pas été publiée à grande échelle, les auteurs de la revue systématique concluent que ces résultats prometteurs mériteraient d'être explorés [69].

\subsubsection{Une gestion optimale de la charge de travail} Une gestion optimale de la charge de travail imposée aux athlètes, avec un équilibre entre phases d'entraînements/compétitions à haute intensité et phases de repos, apparaît importante. En période compétitive chez des sportifs non habitués à ce type de travail, éviter les exercices à haute intensité comprenant des contractions excentriques en positions d'allongement musculaire maximal semble cohérent : les DOMS associés aux déficits transitoires de force auront pour conséquence, en plus de réduire les performances, de rendre la musculature plus vulnérable aux blessures [43].

\subsubsection{La correction d'une mauvaise gestuelle sportive} La correction d'une mauvaise gestuelle sportive pourrait aussi représenter un outil susceptible d'éviter certaines blessures musculaires. Par exemple, une course en cycle postérieur qui se caractérise notamment par un pied qui monte haut à la fesse et des mouvements de jambes qui se déroulent globalement dans un secteur postérieur au centre de gravité mettent une tension plus importante sur le droit fémoral du quadriceps, ce qui pourrait favoriser sa déchirure. Dans cette même optique, une étude récente [70] a analysé la biomécanique de sprint de footballeurs semi-professionnels lors du retour sur terrain après lésion des ischiojambiers et conclut que celle-ci reste perturbée malgré l'autorisation de reprise sportive. L'utilisation d'outils d'analyse biomécanique, tant en prévention primaire qu'après blessure, pourrait donc s'avérer particulièrement intéressante. 
Enfin, selon certains experts [43], la réalisation régulière d'exercices de core stability (que l'on pourrait traduire par qualités de gainage), susceptibles de réduire l'incidence de lésions ligamentaires des membres inférieurs, pourrait aussi avoir un impact positif sur la survenue de lésions musculaires. Le shoot en football ou en rugby, geste qui provoque régulièrement des lésions du droit fémoral, est un exemple intéressant. Lorsqu'un sportif shoote dans un ballon, le tronc est incliné vers l'arrière (de 118 à $128^{\circ}$ ) et ce déplacement postérieur de la partie supérieure du corps génère un degré d'allongement conséquent du droit fémoral. De plus, le caractère asymétrique d'un tir induit également des forces de rotation importantes qui majorent les tensions sur ce muscle. Les qualités neuromusculaires du complexe tronc-bassin-hanches devraient permettre, par un contrôle postural adéquat, de réduire ces contraintes et de placer le droit fémoral dans une position moins risquée.

\section{Conclusion}

Les principaux facteurs de risque intrinsèques des lésions musculaires des membres inférieurs sont essentiellement un premier épisode lésionnel, l'âge et la présence d'un déséquilibre musculaire. Un manque de souplesse contribue probablement à favoriser la survenue d'une blessure musculaire, mais l'évidence scientifique ne peut qu'être qualifiée de moyenne. Les facteurs de risque extrinsèques sont essentiellement le type de sport pratiqué, un échauffement inadapté, une charge de travail mal équilibrée ainsi que la pratique de l'activité en compétition. Certaines différences apparaissent cependant en fonction de la localisation de l'atteinte musculaire. D'un point de vue préventif, la correction de déséquilibres musculaires ainsi que la pratique d'exercices de renforcement à haute intensité en mode excentrique apparaissent fondamentaux. La pratique d'étirements représente certainement un élément intéressant mais l'évidence scientifique reste faible. Enfin, des études ultérieures sont nécessaires pour confirmer l'intérêt de la thérapie manuelle ou d'exercices de gainages.

\section{Déclaration de liens d'intérêts}

Les auteurs déclarent ne pas avoir de liens d'intérêts.

\section{Références}

[1] Ekstrand J, Hagglund M, Walden M. Epidemiology of muscle injuries in professional football (soccer). Am J Sports Med 2011;39(6):1226-32.

[2] Edouard P, Feddermann-Demont N, Alonso JM, Branco P, Junge A. Sex differences in injury during top-level international athletics championships: surveillance data from 14 championships between 2007 and 2014. Br J Sports Med 2015;49(7):472-7, http://dx.doi.org/10.1136/bjsports-2014-094316 [Epub 2015 Jan 24].

[3] Mueller-Wohlfahrt HW, Haensel L, Mithoefer K, Ekstrand J, English B, McNally S, et al. Terminology and classification of muscle injuries in sport: the Munich consensus statement. $\mathrm{Br} \mathrm{J}$ Sports Med 2013;47(6):342-50.

[4] Eirale C, Farooq A, Smiley FA, Tol JL, Chalabi H. Epidemiology of football injuries in Asia: a prospective study in Qatar. J Sci Med Sport 2013;16(2):113-7.
[5] Hagglund M, Walden M, Ekstrand J. Injuries among male and female elite football players. Scand J Med Sci Sports 2009;19(6):819-27.

[6] Brooks JH, Fuller CW, Kemp SP, Reddin DB. Epidemiology of injuries in English professional rugby union: part 1 match injuries. Br J Sports Med 2005;39(10):757-66.

[7] Croisier JL. Factors associated with recurrent hamstring injuries. Sports Med 2004;34(10):681-95.

[8] Orchard J, Seward H. Epidemiology of injuries in the Australian Football League, seasons 1997-2000. Br J Sports Med 2002;36(1):39-44.

[9] Cross TM, Gibbs N, Houang MT, Cameron M. Acute quadriceps muscle strains: magnetic resonance imaging features and prognosis. Am J Sports Med 2004;32(3):710-9.

[10] Brophy RH, Wright RW, Powell JW, Matava MJ. Injuries to kickers in American football: the National Football League experience. Am J Sports Med 2010;38(6):1166-73, http://dx.doi.org/10.77/0363546509357836 [Epub 2010 Mar 2].

[11] Orchard J, James T, Alcott E, Carter S, Farhart P. Injuries in Australian cricket at first class level 1995/1996 to 2000/2001. Br J Sports Med 2002;36(4):270-4 [discussion 5].

[12] Molsa J, Airaksinen O, Nasman O, Torstila I. Ice hockey injuries in Finland. A prospective epidemiologic study. Am J Sports Med 1997;25(4):495-9.

[13] Tyler TF, Nicholas SJ, Campbell RJ, McHugh MP. The association of hip strength and flexibility with the incidence of adductor muscle strains in professional ice hockey players. Am J Sports Med 2001;29(2):124-8.

[14] van Mechelen W, Hlobil H, Kemper HC, Voorn WJ, de Jongh HR. Prevention of running injuries by warm-up, cool-down, and stretching exercises. Am J Sports Med 1993;21(5):711-9.

[15] Orchard J, Best TM, Verrall GM. Return to play following muscle strains. Clin J Sport Med 2005;15(6):436-41.

[16] Brooks JH, Fuller CW, Kemp SP, Reddin DB. Incidence, risk, and prevention of hamstring muscle injuries in professional rugby union. Am J Sports Med 2006;34(8):1297-306.

[17] Ekstrand J, Hagglund M, Walden M. Injury incidence and injury patterns in professional football: the UEFA injury study. $\mathrm{Br} \mathrm{J}$ Sports Med 2011;45(7):553-8.

[18] Hagglund M, Walden M, Ekstrand J. Previous injury as a risk factor for injury in elite football: a prospective study over two consecutive seasons. Br J Sports Med 2006;40(9):767-72.

[19] Fulton J, Wright K, Kelly M, Zebrosky B, Zanis M, Drvol C, et al. Injury risk is altered by previous injury: a systematic review of the literature and presentation of causative neuromuscular factors. Int J Sports Phys Ther 2014;9(5):583-95.

[20] Arnason A, Sigurdsson SB, Gudmundsson A, Holme I, Engebretsen L, Bahr R. Risk factors for injuries in football. Am J Sports Med 2004;32(Suppl. 1):5S-16S.

[21] Henderson G, Barnes CA, Portas MD. Factors associated with increased propensity for hamstring injury in English Premier League soccer players. J Sci Med Sport 2010;13(4):397-402.

[22] Gabbe BJ, Bennell KL, Finch CF, Wajswelner H, Orchard JW. Predictors of hamstring injury at the elite level of Australian football. Scand J Med Sci Sports 2006;16(1):7-13.

[23] Woods C, Hawkins RD, Maltby S, Hulse M, Thomas A, Hodson A. The Football Association Medical Research Programme: an audit of injuries in professional football-analysis of hamstring injuries. Br J Sports Med 2004;38(1):36-41.

[24] Gabbe BJ, Bennell KL, Finch CF. Why are older Australian football players at greater risk of hamstring injury? J Sci Med Sport 2006;9(4):327-33.

[25] Prior M, Guerin M, Grimmer K. An evidence-based approach to hamstring strain injury: a systematic review of the literature. Sports Health 2009;1(2):154-64.

[26] Croisier JL, Ganteaume S, Binet J, Genty M, Ferret JM. Strength imbalances and prevention of hamstring injury in 
professional soccer players: a prospective study. Am J Sports Med 2008;36(8):1469-75.

[27] Dauty M, Potiron-Josse M, Rochcongar P. Consequences and prediction of hamstring muscle injury with concentric and eccentric isokinetic parameters in elite soccer players. Ann Readapt Med Phys 2003;46(9):601-6.

[28] Croisier JL, Forthomme B, Namurois MH, Vanderthommen M, Crielaard JM. Hamstring muscle strain recurrence and strength performance disorders. Am J Sports Med 2002;30(2):199-203.

[29] Yeung SS, Suen AM, Yeung EW. A prospective cohort study of hamstring injuries in competitive sprinters: preseason muscle imbalance as a possible risk factor. $\mathrm{Br} \mathrm{J}$ Sports Med 2009;43(8):589-94.

[30] Bradley PS, Portas MD. The relationship between preseason range of motion and muscle strain injury in elite soccer players. J Strength Cond Res 2007;21(4):1155-9.

[31] Witvrouw E, Danneels L, Asselman P, D’Have T, Cambier D. Muscle flexibility as a risk factor for developing muscle injuries in male professional soccer players. A prospective study. Am J Sports Med 2003;31(1):41-6.

[32] Bennell K, Tully E, Harvey N. Does the toe-touch test predict hamstring injury in Australian Rules footballers? Austr J Physiother 1999;45(2):103-9.

[33] Orchard J, Marsden J, Lord S, Garlick D. Preseason hamstring muscle weakness associated with hamstring muscle injury in Australian footballers. Am J Sports Med 1997;25(1):81-5.

[34] Freckleton G, Pizzari T. Risk factors for hamstring muscle strain injury in sport: a systematic review and meta-analysis. $\mathrm{Br} \mathrm{J}$ Sports Med 2013;47(6):351-8.

[35] Hennessey L, Watson AW. Flexibility and posture assessment in relation to hamstring injury. $\mathrm{Br} J$ Sports Med 1993;27(4):243-6.

[36] Devlin L. Recurrent posterior thigh symptoms detrimental to performance in rugby union: predisposing factors. Sports Med 2000;29(4):273-87.

[37] Cibulka MT, Rose SJ, Delitto A, Sinacore DR. Hamstring muscle strain treated by mobilizing the sacroiliac joint. Phys Ther 1986;66(8):1220-3.

[38] Opar DA, Williams MD, Shield AJ. Hamstring strain injuries: factors that lead to injury and re-injury. Sports Med 2012;42(3):209-26.

[39] Garrett Jr WE. Muscle strain injuries. Am J Sports Med 1996;24(Suppl. 6):S2-8.

[40] Safran MR, Garrett Jr WE, Seaber AV, Glisson RR, Ribbeck BM. The role of warmup in muscular injury prevention. Am J Sports Med 1988;16(2):123-9.

[41] Hawkins RD, Fuller CW. A prospective epidemiological study of injuries in four English professional football clubs. Br J Sports Med 1999;33(3):196-203.

[42] Heidt Jr RS, Sweeterman LM, Carlonas RL, Traub JA, Tekulve FX. Avoidance of soccer injuries with preseason conditioning. Am J Sports Med 2000;28(5):659-62.

[43] Mendiguchia J, Alentorn-Geli E, Idoate F, Myer GD. Rectus femoris muscle injuries in football: a clinically relevant review of mechanisms of injury, risk factors and preventive strategies. Br J Sports Med 2013;47(6):359-66.

[44] Mair SD, Seaber AV, Glisson RR, Garrett Jr WE. The role of fatigue in susceptibility to acute muscle strain injury. Am J Sports Med 1996;24(2):137-43.

[45] Thelen DG, Chumanov ES, Hoerth DM, Best TM, Swanson SC, Li $\mathrm{L}$, et al. Hamstring muscle kinematics during treadmill sprinting. Med Sci Sports Exerc 2005;37(1):108-14.

[46] Pinniger GJ, Steele JR, Groeller H. Does fatigue induced by repeated dynamic efforts affect hamstring muscle function? Med Sci Sports Exerc 2000;32(3):647-53.

[47] Engebretsen AH, Myklebust G, Holme I, Engebretsen L, Bahr R. Intrinsic risk factors for hamstring injuries among male soccer players: a prospective cohort study. Am J Sports Med 2010;38(6):1147-53.
[48] Hagglund M, Walden M, Magnusson H, Kristenson K, Bengtsson $\mathrm{H}$, Ekstrand J. Injuries affect team performance negatively in professional football: an 11-year follow-up of the UEFA Champions League injury study. Br J Sports Med 2013;47(12): $738-42$.

[49] Hagglund $M$, Walden $M$, Ekstrand J. Risk factors for lower extremity muscle injury in professional soccer: the UEFA Injury Study. Am J Sports Med 2013;41(2):327-35, http://dx.doi.org/10.1177/0363546512470634 [Epub 2012 Dec 21].

[50] Woods C, Hawkins R, Hulse M, Hodson A. The Football Association Medical Research Programme: an audit of injuries in professional football-analysis of preseason injuries. Br J Sports Med 2002;36(6):436-41 [discussion 41].

[51] Orchard JW. Intrinsic and extrinsic risk factors for muscle strains in Australian football. Am J Sports Med 2001;29(3):300-3.

[52] Fousekis K, Tsepis E, Poulmedis P, Athanasopoulos S, Vagenas G. Intrinsic risk factors of non-contact quadriceps and hamstring strains in soccer: a prospective study of 100 professional players. Br J Sports Med 2011;45(9):709-14.

[53] Orchard J. The 'northern bias' for injuries in the Australian Football League. Aust Turfgrass Manag 2000;23:36-42.

[54] Hrysomallis C. Hip adductors' strength, flexibility, and injury risk. J Strength Cond Res 2009;23(5):1514-7.

[55] Ekstrand J, Gillquist J. Soccer injuries and their mechanisms: a prospective study. Med Sci Sports Exerc 1983;15(3):267-70.

[56] Ibrahim A, Murrell GA, Knapman P. Adductor strain and hip range of movement in male professional soccer players. J Orthop Surg 2007;15(1):46-9.

[57] Emery CA, Meeuwisse WH. Risk factors for groin injuries in hockey. Med Sci Sports Exerc 2001;33(9):1423-33.

[58] O'Connor D. Groin injuries in professional rugby league players: a prospective study. J Sports Sci 2004;22(7):629-36.

[59] van der Horst N, Smits DW, Petersen J, Goedhart EA, Backx FJ. The preventive effect of the Nordic hamstring exercise on hamstring injuries in amateur soccer players: a randomized controlled trial. Am J Sports Med 2015;43(6):1316-23.

[60] Petersen J, Thorborg K, Nielsen MB, Budtz-Jorgensen E, Holmich P. Preventive effect of eccentric training on acute hamstring injuries in men's soccer: a cluster-randomized controlled trial. Am J Sports Med 2011;39(11):2296-303.

[61] Delvaux F, Rochcongar P, Bruyere O, Bourlet G, Daniel C, Diverse $P$, et al. Return-to-play criteria after hamstring injury: actual medicine practice in professional soccer teams. J Sports Sci Med 2014;13(3):721-3.

[62] Tyler TF, Nicholas SJ, Campbell RJ, Donellan S, McHugh MP. The effectiveness of a preseason exercise program to prevent adductor muscle strains in professional ice hockey players. Am J Sports Med 2002;30(5):680-3.

[63] Kaux JF, Hody S, Delvaux F, Forthomme B, Le Goff C, Massart $\mathrm{N}$, et al. The eccentric intervention for prevention: muscle and tendon aspects. Eur J Sports Med 2014;1(2):37-46.

[64] Arnason A, Andersen TE, Holme I, Engebretsen L, Bahr R. Prevention of hamstring strains in elite soccer: an intervention study. Scand J Med Sci Sports 2008;18(1):40-8.

[65] Brughelli M, Mendiguchia J, Nosaka K, Idoate F, Arcos AL, Cronin J. Effects of eccentric exercise on optimum length of the knee flexors and extensors during the preseason in professional soccer players. Phys Ther Sport 2010;11(2):50-5.

[66] Pope RP, Herbert RD, Kirwan JD, Graham BJ. A randomized trial of preexercise stretching for prevention of lower-limb injury. Med Sci Sports Exerc 2000;32(2):271-7.

[67] McHugh MP, Cosgrave $\mathrm{CH}$. To stretch or not to stretch: the role of stretching in injury prevention and performances. Scand J Med Sci Sports 2010;20(2):169-81.

[68] McCall A, Davison M, Andersen TE, Beasley I, Bizzini $M$, Dupont $G$, et al. Injury prevention strategies 
at the FIFA 2014 World Cup: perceptions and practices of the physicians from the 32 participating national teams. $\mathrm{Br} \mathrm{J}$ Sports Med 2015;49(9):603-8, http://dx.doi.org/10.1136/bjsports-2015-094747.

[69] Goldman EF, Jones DE. Interventions for preventing hamstring injuries. Cochrane Database Syst Rev 2010;(1):CD006782, http://dx.doi.org/10.1002/14651858.CD006782.pub2.
[70] Mendiguchia J, Samozino P, Martinez-Ruiz E, Brughelli M, Schmikli S, Morin JB, et al. Progression of mechanical properties during on-field sprint running after returning to sports from a hamstring muscle injury in soccer players. Int J Sports Med 2014;35(8):690-5. 